\title{
More than a simple crystal
}

\author{
The expanding realm of diamond and related superhard materials requires understanding their structural \\ complexity and the correlation with synthesis conditions for potential properties engineering.
}

$\mathrm{C}$ ommonly known as the hardest natural material on Earth, diamond takes billions of years to form under the extreme temperature and pressure conditions deep beneath the surface. With the name derived from the Greek 'adamas' meaning 'untameable', diamond is reminiscent of hard, pure and clear crystals popularly used in jewellery. Yet the majority of mined diamonds, or those artificially synthesized, do not reach gem quality due to crystalline imperfections, but all have uses as an important engineering material in industry. Possessing outstanding hardness, thermal conductivity and chemical inertness, they are idea for cutting, polishing or grinding, whereas diamonds with favourable optical, electrically insulating and thermally conducting properties are found in optics and optoelectronics applications. Also worth noting is the diamond anvil cell, a device widely used in high-pressure research.

Diamonds with different degrees of crystallinity can be synthesized in different ways to meet the quality and cost requirements of various applications. Large-size single crystals, for instance, have been grown under high-pressure and high-temperature (HPHT) conditions using catalyst solvents. Mono-, poly- or nanocrystalline diamond films can be deposited on different substrates by chemical vapour-phase deposition in the presence of atomic hydrogen. Carbon precursors can also be directly converted into diamond polycrystals under static HPHT mimicking the natural formation conditions in the Earth, or under impact events including detonation or shock compression resembling the formation process of meteoritic diamonds.

The manufacturing process and the carbon sources used determine the type and amount of crystal defects present in the diamonds, which ultimately influence their mechanical and physical properties - for instance, hardness, toughness, as well as electronic, thermal and optical properties. The structure of a perfect diamond crystal consists of carbon atoms covalently bonded in a tetrahedral network in $s p^{3}$ hybridization, arranged in long-range order - a structure called the diamond cubic lattice. However, the occurrence of grain boundaries, reflection or rotation twins, stacking faults introducing hexagonality, or graphite-like

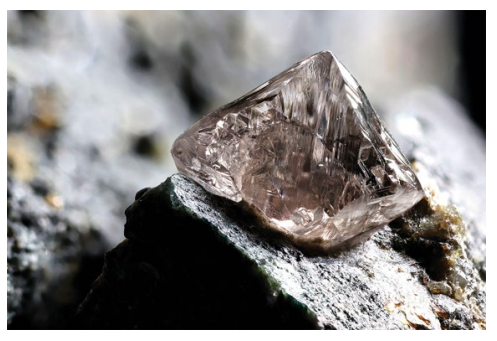

Credit: Björn Wylezich/Alamy Stock Photo

$s p^{2}$-bonded domains, accompanied with intergrowth and mixture of all these defect-induced nanostructures, substantially increases the structural complexity. In this issue, a Comment by Péter Németh and colleagues classifies the nanostructures reported in diamond materials, and maps them with other common crystalline carbon materials in an energy-volume phase space that provides at a glance an overview of the relationship between diamond structures and other carbon polymorphs. The mechanical, thermal and optoelectronic properties associated with the presence of these nanostructures are outlined, providing prospects for future development of diamond-related materials based on nanostructure engineering, which they note is currently still in its infancy.

Beyond crystalline diamonds, various amorphous carbon materials containing mixed $s p^{2}-s p^{3}$ or purely $s p^{3}$ bonding ${ }^{1}$ also show excellent diamond-like mechanical properties. The fraction of $s p^{3}$ bonding and atomic configuration would naturally affect the density and hardness of the resulting materials, while it is not an easy task to characterize different amorphous structures, that is, the local atomic order they possess despite lacking long-range order. Similarly to metallic glasses whose properties are strongly affected by short-range and medium-range order ${ }^{2}$, the identification of amorphous structures and the understanding of their correlation with macroscopic behaviour may help to design amorphous carbons with stronger or unique performance.

From a theoretical viewpoint, the properties of diamond-like materials and more generally carbon materials can be tuned by regulating the bonding character and their structures from the atomic to micro scale. Diverse nanostructures and allotropes have been observed or predicted in the literature. However, a fine control among these structures is difficult to achieve; especially as many are metastable at ambient pressure and temperature and substantially impacted by synthesis routes which often involve extreme conditions. Moreover, the intermediate structures or hybrids that contain stacking disorders and mixed bonding types are not individual phases, and how to obtain them in usable quantities is yet to be explored. Understanding how these different structures are related, their transformation mechanisms and the transition energy barriers that determine the specific requirements of formation conditions will be essential to master the engineering of diamond-like materials, as commented by Németh and colleagues. Computational methods including machine learning can serve as efficient tools to search for superhard structures and assess their stability and synthesizability, as well as to predict structural evolution and synthesis pathways, although this is challenging for metastable materials that have to consider kinetic barriers and a vast configuration space.

Beyond carbon, other types of elements can be brought into play to form diamond-like compounds showing superhardness and interesting electric/ electronic properties, including small elements like boron, nitrogen and oxygen forming short, strong covalent bonds, as well as transition metal elements like osmium, iridium or rhenium introducing a high density of valence electrons ${ }^{3}$. In diamond and related materials, the variety of bonding and versatility of atomic arrangements lead to high levels of structural complexity. But on the positive side, they also offer a high degree of design freedom for reaching desired properties, which must be controlled on the basis of crucial fundamental understanding on the transformation mechanisms and capability of structural control in practice.

Published online: 20 October 2020 https://doi.org/10.1038/s41563-020-00848-6

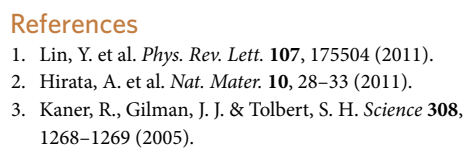

